\title{
Quantitative in situ time-series evaluation of osteoblastic collagen synthesis under cyclic strain using second-harmonic-generation microscopy
}

\author{
Katsuya Sato \\ Oki Matsubara \\ Eiji Hase \\ Takeo Minamikawa \\ Takeshi Yasui
}




\title{
Quantitative in situ time-series evaluation of osteoblastic collagen synthesis under cyclic strain using second-harmonic-generation microscopy
}

\author{
Katsuya Sato, ${ }^{\mathrm{a}, \star}$ Oki Matsubara, ${ }^{\mathrm{a}}$ Eiji Hase,${ }^{\mathrm{b}}$ Takeo Minamikawa, ${ }^{\mathrm{a}}$ and Takeshi Yasui ${ }^{\mathrm{a}}$ \\ ${ }^{a}$ Tokushima University, Graduate School of Technology, Industrial and Social Sciences, Tokushima City, Tokushima, Japan \\ bJapan Synchrotron Radiation Research Institute, Research and Utilization Division, Sayo, Hyogo, Japan
}

\begin{abstract}
The aim of this study is to evaluate the osteoblastic collagen synthesis under mechanical stimulation using second-harmonic-generation (SHG) microscopy. We apply SHG microscopy to monitor the collagen fibers synthesized by osteoblast-like cells (MC3T3-E1) without the need for fixation and staining. To quantitatively evaluate the influence of mechanical stimulation on osteoblastic collagen synthesis, we compare SHG images of osteoblast-synthesized collagen fibers with and without a cyclic stretch stimulus applied using a lab-made stretching device. We acquire SHG images every 7 days for 3 weeks at different stimulus conditions $(5 \mathrm{~min} /$ day and $3 \mathrm{~h} /$ day with a strain magnitude of $5 \%$ and a frequency of $0.5 \mathrm{~Hz}$ ). Image analysis of the average SHG intensity indicates that the amount of osteoblastic collagen synthesis is significantly enhanced by the cyclic stretch compared with the nonstretched condition, while there is no significant difference between the two mechanical stimulation conditions. Furthermore, the maturity of the collagen fibers in the early stage of bone formation is not affected by the mechanical stimulation. The results can be used in bone regenerative medicine to apply feedback control of collagen synthesis by artificial stimulation. $\odot$ The Authors. Published by SPIE under a Creative Commons Attribution 4.0 Unported License. Distribution or reproduction of this work in whole or in part requires full attribution of the original publication, including its DOI. [DOI: 10.1117/1.JBO.24.3.031019]
\end{abstract}

Keywords: second-harmonic-generation; collagen; osteoblast; mechanical stimulation; bone regenerative medicine.

Paper 180368SSRR received Jun. 19, 2018; accepted for publication Dec. 11, 2018; published online Jan. 11, 2019.

\section{Introduction}

Bone cells, such as osteoblasts, osteoclasts, and osteocytes, play important roles in bone metabolism, such as bone formation and resorption. ${ }^{1}$ Bone matrix consists of hydroxyapatite and type I collagen, and the latter occupies $\sim 90 \%$ of bone matrix. However, as bone metabolism is quite slow, it is difficult for elderly to heal damaged bone or increase the sufficient amount of bone during a short period. ${ }^{2}$ Therefore, there is a considerable need for bone tissue engineering and regenerative medicine to locally replace damaged bone in older patients.

For the regenerated bone, it is essential for the osteoblasts to effectively generate the collagen fibers. It has been shown that the amount of collagen produced by osteoblasts can be significantly increased by applying a mechanical stimulus. ${ }^{3-6}$ To apply a suitable mechanical stimulation for collagen fiber production, it is necessary to noninvasively monitor the collagen dynamics under mechanical stimuli. Conventionally, staining methods have been widely used to visualize collagen fibers in the tissue. ${ }^{7,8}$ However, invasiveness due to fixation and staining makes it difficult to apply this method for in situ monitoring of collagen fiber production by osteoblasts over time. Recently, second-harmonic-generation (SHG) microscopy has been used to visualize the collagen fibers in situ without the need for fixation and staining. ${ }^{9,10}$ SHG light is generated specifically from collagen molecules in biological tissues. Additionally, SHG microscopy has high selectivity and good image contrast for collagen molecules as well as high spatial resolution, optical three-dimensional (3-D) sectioning, moderate penetration, and

*Address all correspondence to Katsuya Sato, E-mail: katsuyas@tokushimau.ac.jp is minimally invasive. Previously, we demonstrated in situ time-series SHG imaging of collagen fibers synthesized by osteoblasts. ${ }^{11}$ However, this experiment was performed under a standing culture condition without a mechanical stimulus.

In this work, we performed in situ time-series SHG imaging of collagen fibers synthesized by osteoblasts under cyclic stretch using a lab-made cell-stretching device. We then quantitatively evaluated the production of collagen fibers by image analysis of the SHG microscopy.

\section{Materials and Methods}

\subsection{Cell Culture}

MC3T3-E1 osteoblast-like cells (Riken BRC, Tsukuba, Japan) were precultured in alpha-minimum essential medium ( $\alpha$-MEM) (Nacalai tesque, Kyoto, Japan) containing 10\% fetal bovine serum (FBS) (Nacalai tesque, Kyoto, Japan) and were maintained at $37^{\circ} \mathrm{C}$ and $5 \% \mathrm{CO}_{2}$ in a humidified incubator until subconfluent. Cells were then seeded onto a polydimethylsiloxane (PDMS) rubber chamber (STB-CH-04, STREX, Osaka, Japan) coated with fibronectin (Wako, Osaka, Japan) at a density of $5.0 \times 10^{4}$ cells $/ \mathrm{cm}^{2}$ and cultured in the culture medium that contains $\alpha$-MEM supplemented with $10 \%$ FBS, $1 \%$ penicillin-streptomycin (Nacalai tesque, Kyoto, Japan). MC3T3$\mathrm{E} 1$ is an osteoblast precursor cell line. We added an osteoblast-inducer reagent to the culture medium (composition of $1 \%$ ascorbic acid, $0.2 \%$ hydrocortisone, and $2 \% \quad \beta$ Glycerophosphate) (TaKaRa, Shiga, Japan) to differentiate cells into osteoblast-like cell and to maintain the differentiated state of cell during experiment. Cells were statically incubated 
for 1 day before stretch application to ensure sufficient cell adhesion. The culture medium was exchanged semiweekly.

\subsection{Application of Mechanical Stimuli}

To apply stretch stimulus to the cells, we developed a custom cell-stretching device as shown in Fig. 1. The device consists of a stepping motor and a ball screw. The rotational motion of the stepping motor is transmitted into linear reciprocating motion by the ball screw. The PDMS chamber with the cells was mounted on the stretching device. The entire device was put into the $\mathrm{CO}_{2}$ incubator during the experimental period. Cell culture periods were set to 1,2 , and 3 weeks. During these periods, stretch stimulus was applied to the cells for $5 \mathrm{~min} /$ day or $3 \mathrm{~h} /$ day with $5 \%$ strain magnitude and $0.5 \mathrm{~Hz}$ loading frequency. The cells cultured statically (no stretch application) in the PDMS chamber were defined as the control group. The number of samples in each stretch condition group is three.

\subsection{Collagen Staining and Observation}

To compare the image obtained using conventional collagen staining method and the image obtained by our SHG microscopy setup, we performed another experiment. Cells were fixed at 1 and 2 weeks culture periods with and without stretching stimulation $(1 \mathrm{~h} /$ day, $5 \%$ strain magnitude, and $0.5-\mathrm{Hz}$ frequency) using $4 \%$ paraformaldehyde, and stained with the collagen stain kit (TaKaRa, Shiga, Japan), which contains sirius red dye. Stained collagen was observed using the phase contrast inverted microscope (CKX-41, Olympus, Tokyo, Japan) with $\times 4$ dry objective lens and recorded with the digital camera (DP71, Olympus, Tokyo, Japan). Obtained RGB color images were converted into cyan, magenta, yellow, and black (CMYK) four color model. We assessed the depth of sirius red staining color using the key plate value of CMYK image.

\subsection{Experimental Setup for Second-Harmonic- Generation Microscopy}

We used SHG microscopy to evaluate the amount and maturity of collagen fibers synthesized by the osteoblasts as shown in Fig. 2. The optical setup of the SHG microscope was essentially the same as was previously reported. ${ }^{11} \mathrm{~A}$ mode-locked Ti:Sapphire laser light (pulse duration at the focal point of $19 \mathrm{fs}$, center wavelength of $787 \mathrm{~nm}$, repetition frequency of 81.8 MHz, laser power of $20 \mathrm{~mW}$ measured at the optical path which guides laser to the microscope) is focused on the sample by an objective lens for water immersion (NA $=0.90$, $\mathrm{WD}=2 \mathrm{~mm}$ ). The laser spot was scanned in a two-dimensional plane on the sample surface using galvano mirrors with relay lenses. The SHG signal was collected by the photo multiplier tube (PMT) (Photon counting head, H8259, Hamamatsu photonics K. K., Shizuoka, Japan), which was mounted on backward to the sample. In all experiments, PMT detector gain

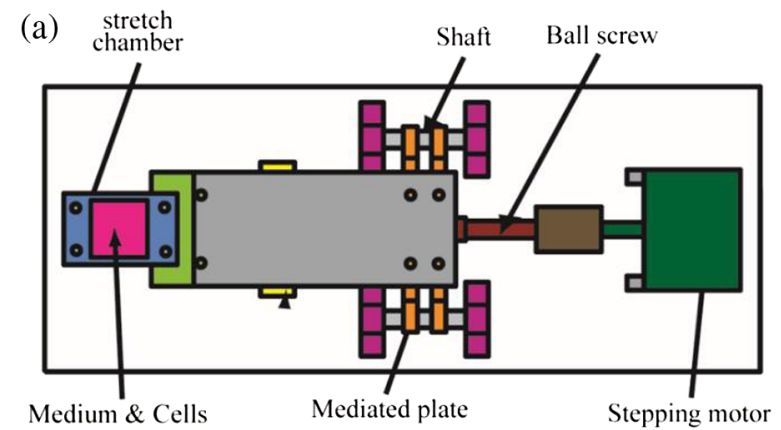

(b)

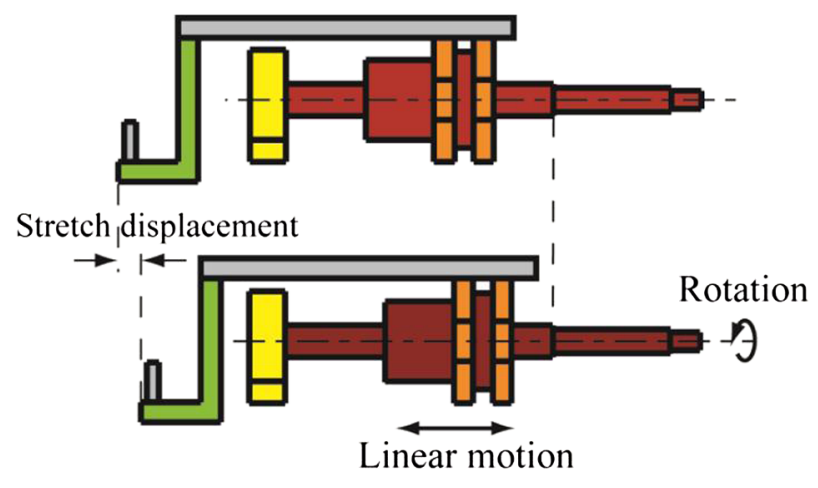

(c)

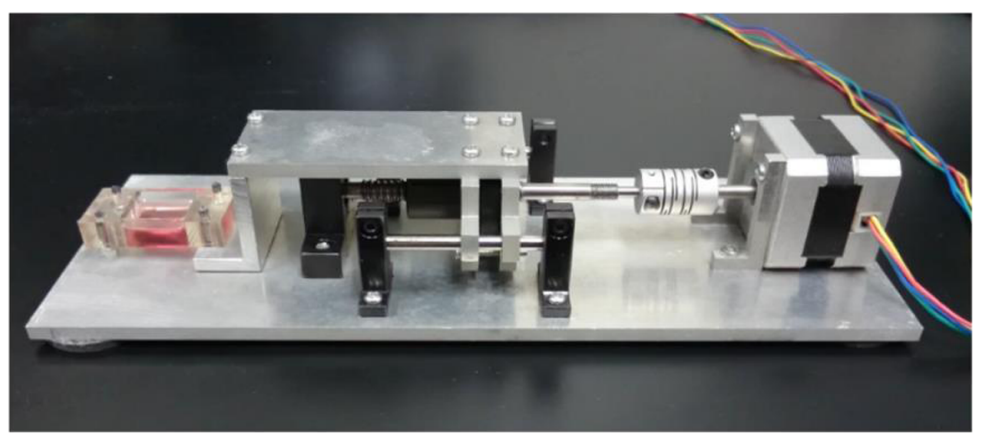

Fig. 1 (a) Schematic of the cell stretch device, (b) mechanism to convert rotational motion into linear motion, and (c) photograph of the cell stretch device. 

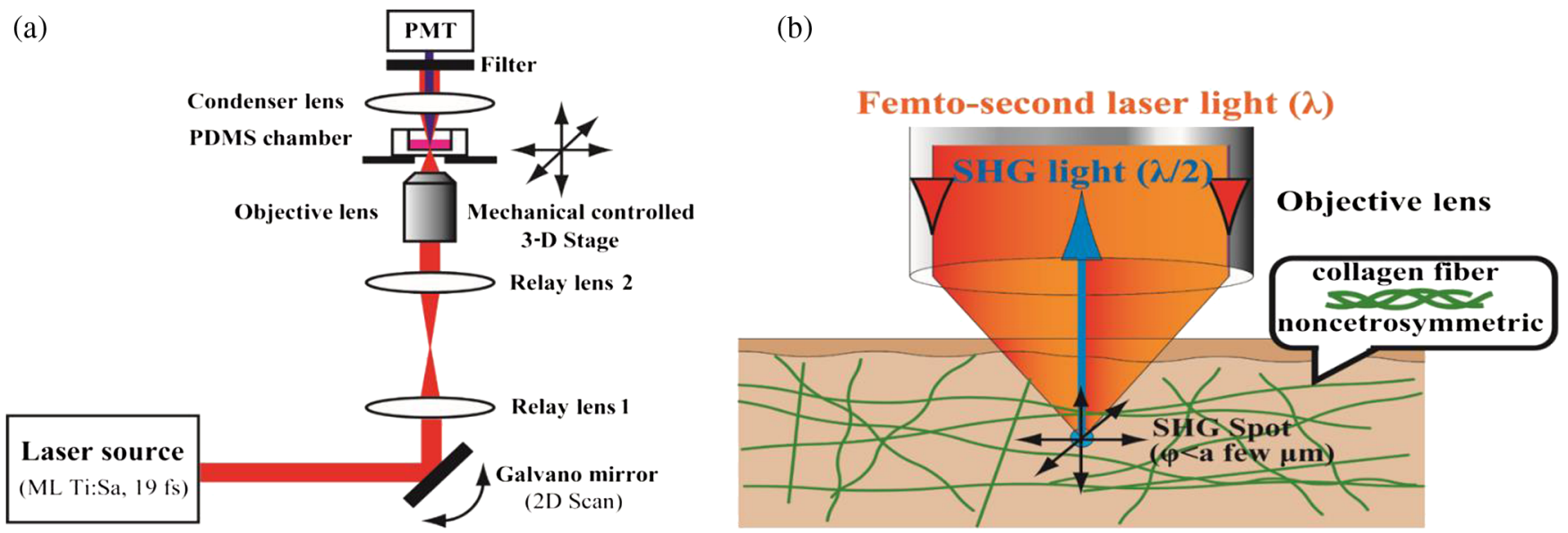

Fig. 2 (a) SHG microscopy experimental setup. The light source is an ML-Ti:S laser and its pulse width is $18.9 \mathrm{fs}$ at the focal spot, and laser power is $20 \mathrm{~mW}$. The laser light is scanned two-dimensionally by Galvano mirrors ( $256 \times 256$ pixels), and the scanning area is expanded by sequential image acquisition using a mechanical control stage. The total image size is $1040 \mu \mathrm{m} \times 1040 \mu \mathrm{m}$ and the total number of pixels is $1,048,576(256 \times 256 \times 16)$. (b) Mechanism of occurring SHG light. The SHG light occurs only from noncentrosymmetric materials (e.g., collagen fibers). The wavelength of the incident light is converted to half wavelength by entering the collagen fiber.

was set constant. The scanning area was $260 \mu \mathrm{m} \times 260 \mu \mathrm{m}$ with a 256-pixel resolution in each direction. Scanning rate was also set constant for all experiments. The scanning area was expanded to include 16 total images acquired sequentially with an electromechanically controlled stage, resulting in a total imaging area of $1040 \mu \mathrm{m} \times 1040 \mu \mathrm{m}$ comprising $1,048,576$ total pixels $(256 \times 256 \times 16)$.

\subsection{Statistics}

We used Student's $t$-test to evaluate the effect of mechanical stimulation on osteoblastic collagen synthesis. We assumed a statistical significance threshold of 0.05 for all experimental data.

\section{Results}

\subsection{Comparison of Conventional Collagen Staining Image and Second-Harmonic-Generation Microscopy Image}

Figure 3 shows the phase contrast images of Sirius red staining collagen synthesized by MC3T3-E1 cells in culturing periods of 1 and 2 weeks with and without stretching stimulation. Figure 4 shows SHG microscopy images of cells that were fixed with $4 \%$ paraformaldehyde. SHG imaging was performed before sirius red staining. The observed area and magnification did not match between the phase contrast image and the SHG image. These images obtained using two kinds of different methods show qualitatively good agreement of distribution of osteoblastic synthesized collagen. Figure 5 shows the histogram of SHG signal intensity in the obtained SHG images. As shown in the graph, according to the culturing time periods and application of stretch stimulation, the peak and foot of right side (meaning high SHG signal intensity) in the histogram curve shift toward the right. Figure 6 shows the correlation diagram between the mean value of SHG signal intensity and the mean key plate value of CMYK image calculated from the sirius red-stained collagen image. There is a good correlation between the SHG microscopy image and conventional sirius red staining

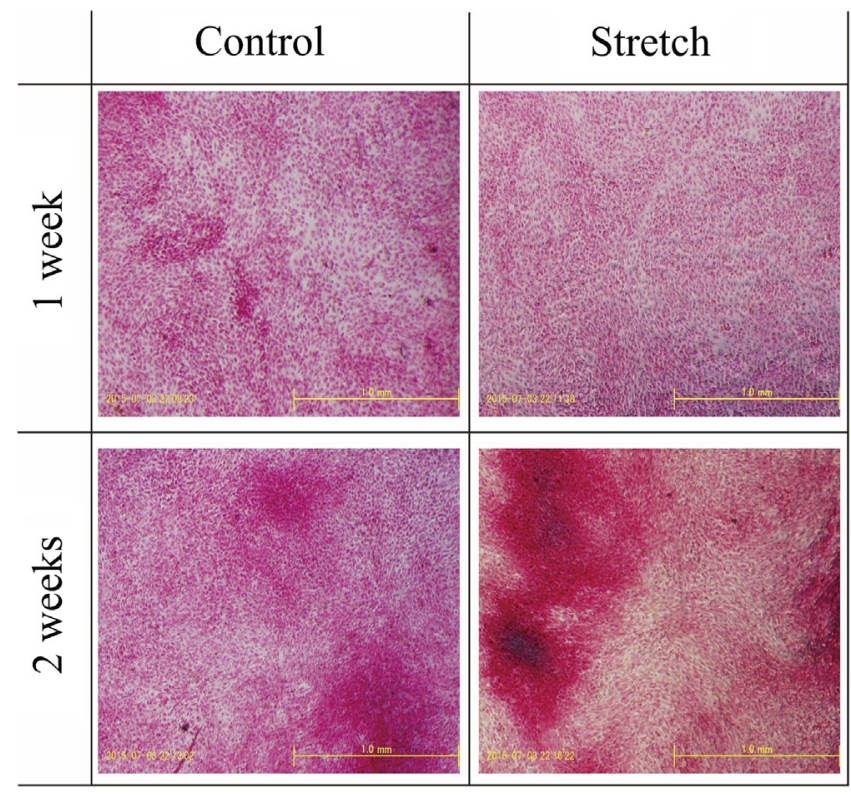

Fig. 3 Osteoblasts synthesized collagen stained by sirius red dye. The area of stained collagen and the color depth of sirius red dye are increased according to the culturing time period and the application of stretching mechanical stimuli.

image. From these results, we concluded that our SHG microscopy setup can be used to assess the amount of osteoblastic collagen synthesis without fixation and staining, and to conduct in situ time-series evaluation.

\subsection{Definition of Matured Fibrillar Collagen Using Second-Harmonic-Generation Signal Intensity}

In Figs. 4(a)-4(c) shows the representative images that reflect the maturation process of osteoblastic-synthesized collagen in vitro obtained by the SHG microscopy. These images are cropped from the entire SHG image, respectively. In the 


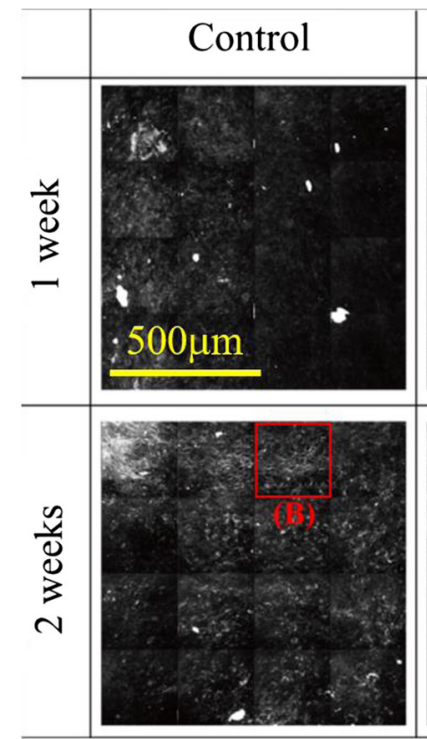

(a)

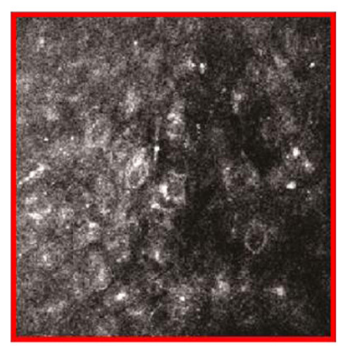

(c)

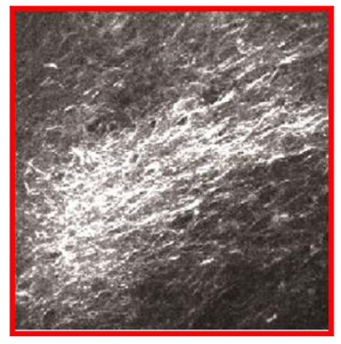

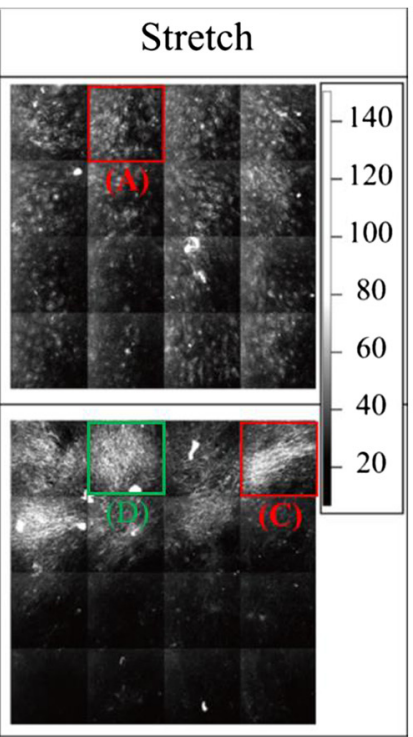

(b)

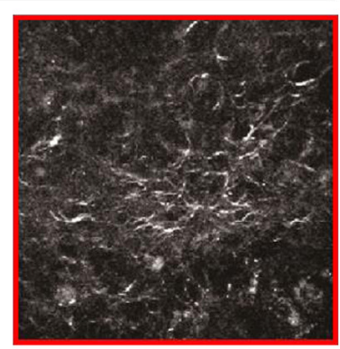

(d)

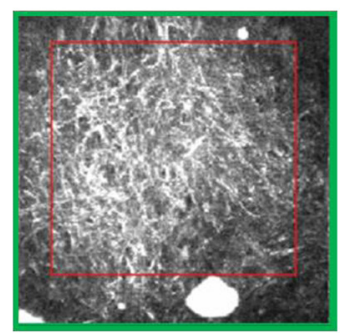

Fig. 4 SHG image of fixed cell and collagen. SHG imaging was conducted before sirius red staining. The observed region was not same with that of resius red stained image presented in the Fig. 3 . The area and the intensities of SHG signal are increased according to the culturing time period and the application of mechanical stimuli. These SHG images show qualitatively good agreement with the sirius red stained image. (a), (b) and (c) Representative magnified images of collagen. Cropped region is indicated by the red rectangle in the entire SHG image. (a) There are distributed mist-like SHG signals, but fibrous structure cannot be observed. This SHG image might detect nonfibrillar collagen synthesized by osteoblastic cells. (b) SHG signals with fragmented thin fibrous structure can be observed. We speculate that is an immature fibrillar collagen. (c) There are thick and dense fibrous structure. We considered that this SHG image detects matured collagen fibers. (d) Representative SHG image that is used to define the threshold SHG signal intensity range. The cropped region shown by the red rectangle in (d) is used to calculate average SHG intensity and standard deviation.

SHG image obtained at 1 week culture period with stretch stimulation [indicated as Fig. 4(a)], there are distributed mistlike SHG signals, but fibrous structure cannot be observed. This SHG image might detect nonfibrillar collagen synthesized by osteoblastic cells. On the other hand, in the SHG image at 2 weeks without stretch stimulation (control sample) [Fig. 4(b)],

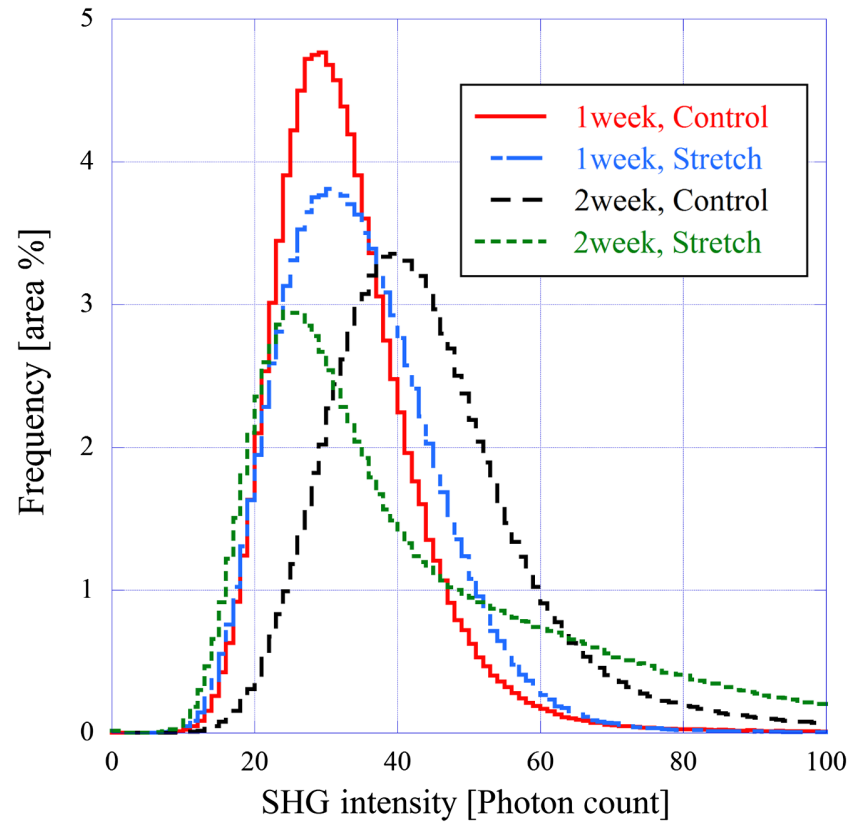

Fig. 5 Histograms of SHG signal intensity of SHG images obtained from fixed cell samples. According to the culturing time periods and application of stretch stimulation, the peak and foot of right side in the histogram curve shift toward the right.

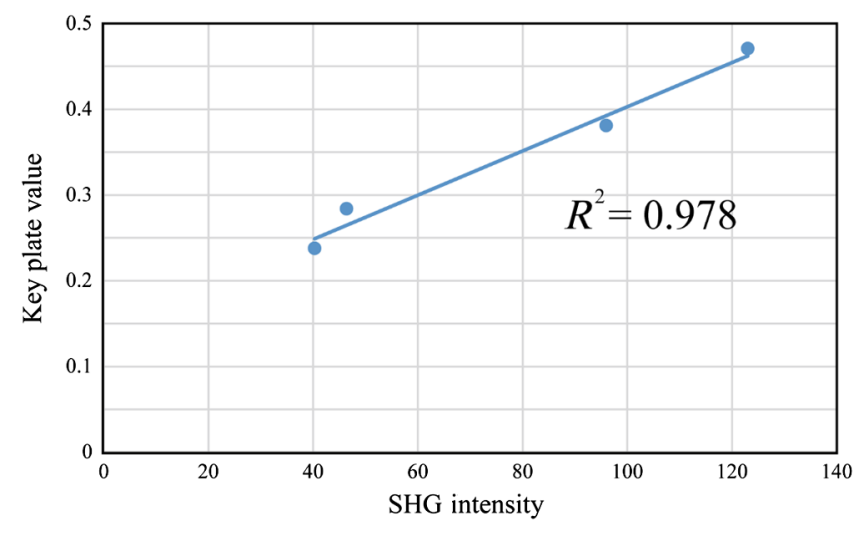

Fig. 6 Correlation diagram between the mean value of SHG signal intensity in fixed cell samples and the mean key plate value of CMYK image calculated from the sirius red stained collagen image. There is a good correlation between the SHG microscopy image and conventional sirius red staining image.

SHG signals with fragmented thin fibrous structure can be observed. We speculate that this is an immature fibrillar collagen. As shown in Fig. 4(c), the SHG image obtained at the 2 weeks culture period with stretch stimulation, there are thick and dense fibrous structures. We considered that this SHG image detects matured collagen fibers.

Based on these observation results, to evaluate the amount of collagen fibers synthesized by cultured osteoblasts under different loading conditions, we set the certain threshold range of SHG signal intensity to detect the matured collagen fibers. Figure 4(d) shows a representative SHG image of well-matured fibrous collagen fibers synthesized by osteoblastic cells in our study. We cropped one region in which matured collagen fibers exist. The histogram of SHG signal intensity in the cropped region is shown in Fig. 7. The mean value of SHG signal 


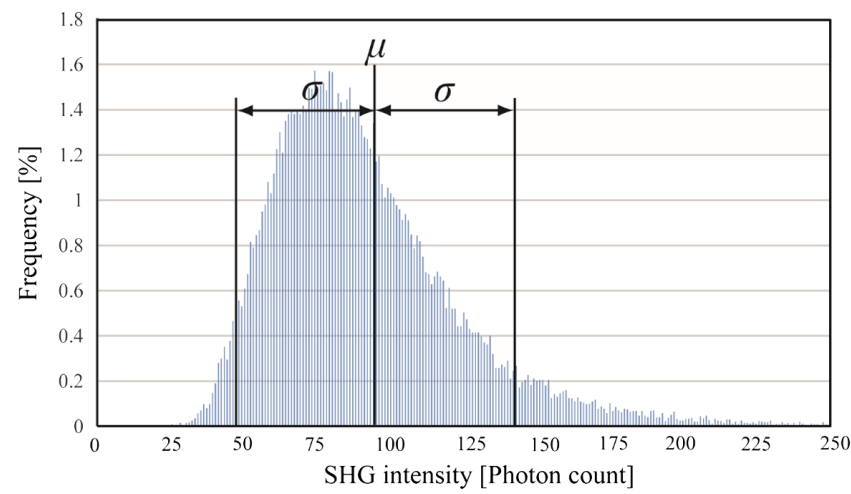

Fig. 7 Histogram of SHG signal intensity calculated from the cropped SHG image, which represents the matured collagen fibers.

intensity from the matured collagen fibers was 90 with a standard deviation of 45 . As this threshold range served as a criterion to determine whether the collagen fibers had matured, we used the values that the number of pixels whose SHG intensity was within the threshold range was divided by the total number of pixels in the SHG image to evaluate the amount of osteoblastic collagen synthesis.

\subsection{In Situ Time-Series Evaluation of Collagen Synthesis Using Second-Harmonic-Generation Microscopy}

We performed SHG imaging of collagen fibers synthesized by cultured osteoblasts under three different conditions (control, stretch for $5 \mathrm{~min} /$ day, and stretch for $3 \mathrm{~h} /$ day) as shown in Fig. 8. The representative magnified images are shown as Figs. 8(a)-8(c), respectively. Cropped regions are indicated by red rectangles in the entire SHG image. The collagen fibers were synthesized and secreted by osteoblasts with the lapse of time. As shown in Fig. 8(a), the nucleus appeared as a dark round area. More importantly, the brightness of the SHG images for each condition increased over time, indicating a temporal change of collagen distribution during the culturing period as shown in Figs. 8(b) and 8(c).

Figure 9 shows the number of pixels that had a measured SHG intensity within the threshold range for each week at each test condition. The graph shows the percentage of pixels

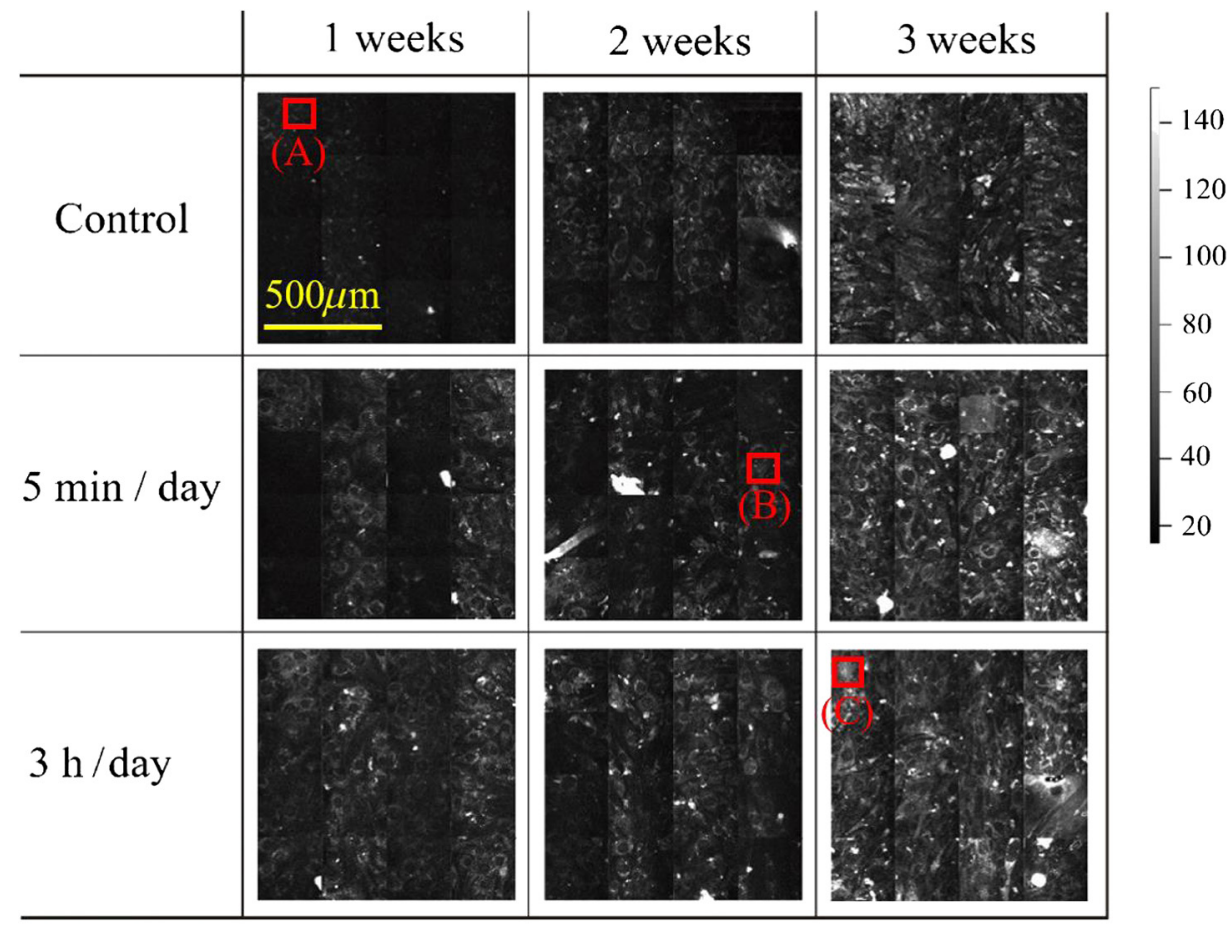

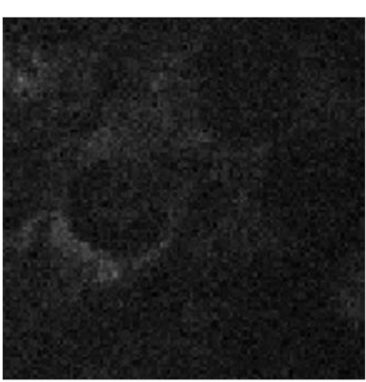

(a) Control lweek

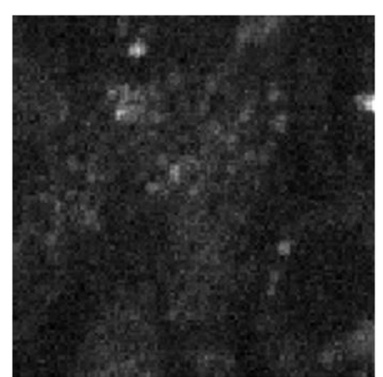

(b) $5 \mathrm{~min} /$ day $2 \mathrm{week}$

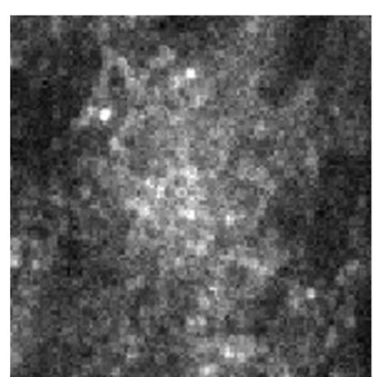

(c) $3 \mathrm{~h} /$ day 3 week

Fig. 8 SHG images of collagen synthesized by osteoblasts at each culturing time periods and each mechanical stimulus conditions. (a), (b), and (c) Magnified representative images of collagen fibers in each culturing time periods and mechanical stimulation conditions. 


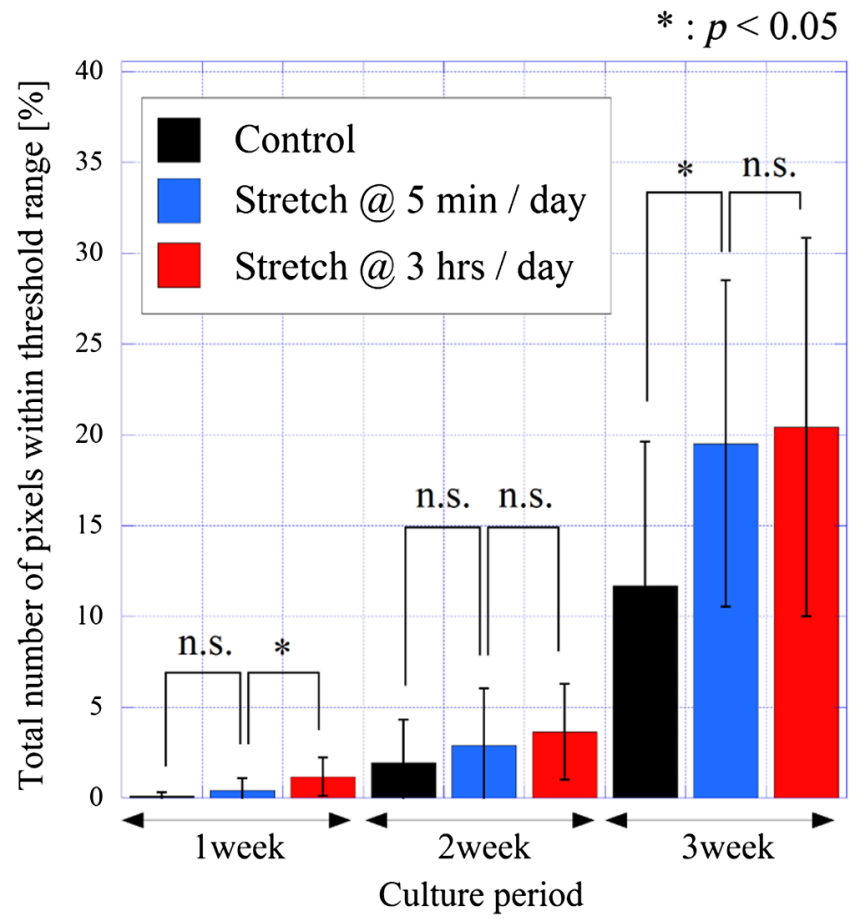

Fig. 9 Total number of pixels within the threshold range (an SHG intensity value of $95 \pm 45$ ), which was considered to matured collagen fibers. These values were normalized by dividing the total number of pixels in the scanned area. Data represent the mean \pm standard deviation. ${ }^{*}$ Symbol indicates a significant difference $(p<0.05$, Student's $t$-test).

within the threshold range calculated from the total number of pixels in each SHG images (1,048,576 pixels). The temporal change in the number of pixels in which SHG signal intensity was within the threshold range for each group was compared. Although the control group exhibited an increase in the number of pixels determined as a collagen fiber, the rate of increase was significantly smaller than those of under stretch conditions. In both stretch condition groups, the number of pixels within the threshold range increased significantly compared with the control group. This result implies that the amount of collagen fibers synthesized by osteoblasts was enhanced by the mechanical stimulation. For all three culture periods (1 to 3 weeks), there was a significant difference between the control group and both stretched groups. However, no significant difference was observed between the two stretch condition groups.

To investigate the differences in collagen fiber maturity more precisely, we calculated the mean SHG intensity for those pixels determined as a collagen fiber. The maturity of collagen fiber includes fiber thickness, degree of cross linking and alignment of microfibrils in the collagen fiber. We hypothesized that more matured collagen fiber exhibits higher SHG signal intensity. Figure 10 shows the mean SHG intensity for the pixels determined as a collagen fiber as a function of time period in three groups. Although the number of pixels which SHG signal intensity was within the threshold range increased steadily during the culture periods (Fig. 9), the mean SHG intensity values for those pixels were almost constant during those three culture periods. This result suggests that the amount of osteoblastic collagen synthesis was steadily increased during the culture period and enhanced by the application of mechanical stimulation,

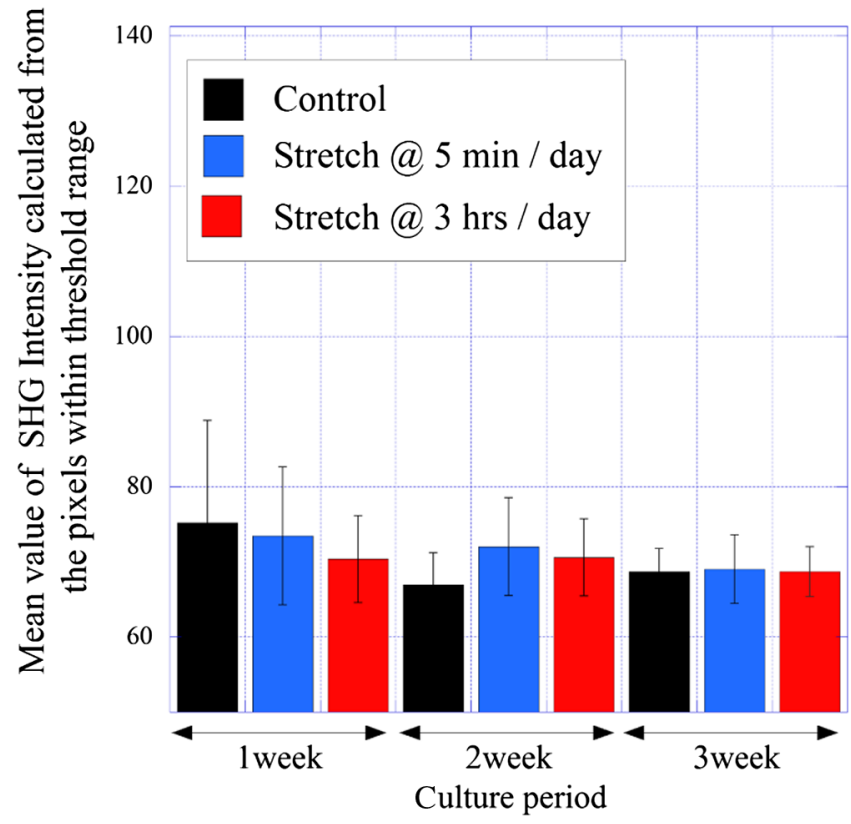

Fig. 10 Relationship between the mean values of the SHG intensity for the pixels within the threshold range. Data represent the mean \pm standard deviation. There was no significant difference between all culturing time periods and all stimulation condition groups.

whereas the maturity of the collagen fibers was not affected by the mechanical stimulation and culturing time period.

\section{Discussion}

Many studies have been conducted to investigate the effect of mechanical stimuli (e.g., cyclic strain or fluid flow) on osteoblastic activities. ${ }^{12,13}$ Kasper et al. ${ }^{14}$ investigated the cellular response to cyclic strain in human-bone-derived cells and reported that the cell proliferation and collagen type I carboxyterminal propeptide (CICP) production were increased by the strain stimulation. Jackson et al. ${ }^{15}$ researched the influence of mechanical strain and fluid flow on the time-course of change of gene expression in osteoblastic cells over several weeks. They reported that the collagen mRNA expression of loaded samples were steadily up-regulated compared with unstimulated samples. However, in their study the collagen synthesis was measured using invasive methods (e.g., Western blotting using antibodies), therefore, the measurement of long-term results was not conducted with the same sample. Our results are consistent with the findings of these studies while using a noninvasive imaging method. In a previous study, we succeeded in visualizing the collagen fibers synthesized by osteoblasts using SHG microscopy without any fixation. ${ }^{11}$ In this study, we succeeded in imaging collagen fibers synthesized by osteoblasts under cyclic stretch stimulation over time. Results showed the amount of collagen fibers produced by the stretched groups was significantly higher compared with the statically cultured group. Our noninvasive in situ monitoring technique for osteoblastic collagen synthesis could be used for the future bone regenerative medicine with artificial control of osteoblastic bone formation using mechanical stimulation. Noninvasive monitoring enables us to adjust and optimize the mechanical stimuli condition to enhance osteoblastic bone forming activity according to the result of assessment based on SHG microscopy images. 
As reported in other studies, osteoblasts increase their collagen synthesis when mechanical stimulation is applied, and our results support this osteoblastic cellular response. Bancroft et al. ${ }^{16}$ and Yan et al. ${ }^{17}$ demonstrated that the osteoblasts change their activities (e.g., cell proliferation and cell secretion) according to the magnitude of the applied mechanical stimulus and the number of stimulation cycles. In contrast, in this study there was no insignificant difference in the amount of collagen fibers between the $5 \mathrm{~min} /$ day and $3 \mathrm{~h}$ /day stretching groups. Bancroft et al. measured the amount of material secreted by osteoblasts under fluid flow for 16 days. They applied the shear fluid flow continuously, while we applied the stretch stimulation to the cells intermittently with defined durations per day. The longest stretching duration in our study is estimated at $63 \mathrm{~h}$, which roughly corresponds to 3 days in the study by Bancroft et al. They reported that there was no significant difference between each flow condition at day three. Moreover, several studies demonstrated that shear fluid flow induced more sensitive cellular response and specific cellular secretion such as No, prostaglandin E2 compared with stretch stimulus. ${ }^{18,19}$ Therefore, the experiment duration used in this study might not be sufficiently long enough to produce significant differences between the two stretch condition groups. Another possibility is the limitation of statistical power in our experimental setup. The number of samples in each experimental condition was 3. It could be insufficient to detect a statistically significant difference between the samples of $5 \mathrm{~min} /$ day stimulation group and $3 \mathrm{~h} /$ day group. We have to conduct further experiments and add the number of samples to discuss about the influence of mechanical stimuli condition to the osteoblastic collagen synthesis.

It was reported that the cell proliferation rate reached a maximum value at a certain stimulation period. ${ }^{20}$ Another study reported that osteoblastic alkaline phosphatase activity exhibited different responses to continuous or discontinuous treatment of hormone. ${ }^{21}$ Cells might be habituated against long-term continuous mechanical stimuli, and there was no significant difference in the amount of collagen fibers between the two stretch condition groups in this study.

We measured the mean value of the SHG intensity for the pixels defined as collagen fiber to evaluate collagen fiber maturity. Although the amount of collagen fibers increased during the culture period in all three groups, the mean values of the SHG intensity calculated from the pixels within the threshold range remained nearly constant. There is a qualitative relationship between the maturity of the high-order structure of collagen fibers and the intensity of the SHG signal; ${ }^{22-24}$ therefore, we speculate that the collagen synthesized by the osteoblasts in the early stage of bone formation did not have as much high-order structure characterized as the collagen in mature bone matrix. Additionally, mechanical stretch stimulation might not enhance the maturation of collagen synthesized by osteoblasts in the early stages of bone formation.

\section{Conclusions}

We conducted in situ time-lapse observations of collagen synthesized by osteoblasts using SHG microscopy without any fixation and staining to evaluate the influence of mechanical stimulation on osteoblastic collagen synthesis. Results showed for all mechanical stimulation condition groups the amount of collagen fibers increased with time. Although there was a significant difference between the stretched groups and the static culture group, there was no significant difference between the two stretch groups with different stretch durations per day. Meanwhile, mean values of the SHG intensity calculated from the pixels that contained mature and well-structured collagen fibers remained nearly constant during the entire culture period. These results suggest that while osteoblastic collagen synthesis is increased by stretch application, mechanical stimulus might not affect the maturity of collagen fibers in the early stage of bone formation.

\section{Disclosures}

The authors have no conflicts of interest directly relevant to the content of this work.

\section{Acknowledgments}

This study was partly supported by JSPS KAKENHI Grant No. 17K0136100. This study has been already published in the proceedings of SPIE 10711, SPIE structured light, Biomedical Imaging and Sensing Conference (BISC) 2018.

\section{References}

1. D. Heinegård and Å. Oldberg, "Structure and biology of cartilage and bone matrix noncollagenous macromolecules," FASEB J. 3, 2042-2051 (1989).

2. M. T. Hannan et al., "Risk factors for longitudinal bone loss in elderly men and women: the Framingham osteoporosis study," J. Bone Miner. Res. 15, 710-720 (2000).

3. L.V. Harter, K.A. Hruska, and R. L. Duncan, "Human osteoblast-like cells respond to mechanical strain with increased bone matrix protein production independent of hormonal regulation," Endocrinology 136, 528-535 (1995).

4. M. C. Meazzini et al., "Osteoblast Cytoskeletal modulation in response to mechanical strain in vivo," J. Orthop Res. 16, 170-180 (1998).

5. A. Ignatius et al., "Tissue engineering of bone: effects of mechanical strain on osteoblastic cells in type I collagen matrices," Biomaterials 26, 311-318 (2005).

6. J. Boada-Lopez et al., "Collagen abundance in mechanically stimulated osteoblast cultures using near infrared microscopy," J. Biomech. 46, 2442-2450 (2013).

7. A. D. McCarthy et al., "Non-enzymatic glycosylation of a type I collagen matrix: effects on osteoblastic development and oxidative stress," BMC Cell Biol. 2, 16 (2001).

8. K. N. Krahn et al., "Fluorescently labeled collagen binding proteins allow specific visualization of collagen in tissues and live cell culture," Anal. Biochem. 350, 177-185 (2006).

9. S. Roth and I. Freund, "Second harmonic generation in collagen," J. Chem. Phys. 70, 1637-1643 (1979).

10. P. J. Campagnola and L. M. Loew, "Second-harmonic imaging microscopy for visualizing biomolecular arrays in cells, tissues and organisms," Nat. Biotechnol. 21, 1356-1360 (2003).

11. E. Hase et al., "In situ time-series monitoring of collagen fibers produced by standing-cultured osteoblasts using a second-harmonic-generation microscope," Appl. Opt. 55, 3261-3267 (2016).

12. S. W. Donahue, C. R. Jacobs, and H. J. Donahue, "Flow-induced calcium oscillations in rat osteoblasts are age, loading frequency, and shear stress dependent," AJP-Cell Physiol. 281, C1635-C1641 (2001).

13. C. Neidlinger-Wilke, H. J. Wilke, and L. Claes, "Cyclic stretching of human osteoblasts affects proliferation and metabolism: a new experimental method and its application," J. Orthop. Res. 12, 70-78 (1994).

14. D. Kasper et al., "Dynamic cell stretching increases human osteoblast proliferation and CICP synthesis but decreases osteocalcin synthesis and alkaline phosphatase activity," J. Biomech. 33, 45-51 (2000).

15. R. A. Jackson et al., "Long-term loading inhibits ERK1/2 phosphorylation and increases FGFR3 expression in MC3T3-E1 osteoblast cells," J. Cell. Physiol. 209, 894-904 (2006).

16. G. N. Bancroft et al., "Fluid flow increases mineralized matrix deposition in $3 \mathrm{D}$ perfusion culture of marrow stromal osteoblasts in a dosedependent manner,' PNAS 99, 12600-12605 (2002). 
17. Y. X. Yan et al., "Mechanical strain regulates osteoblast proliferation through Integrin-mediated ERK activation," PLoS One 7, e35709 (2012).

18. I. Owan et al., "Mechanotransduction in bone: osteoblasts are more responsive to fluid forces than mechanical strain," Am. J. Physiol. 273, C810-C815 (1997).

19. R. Smalt et al., "Induction of NO and prostaglandin E2 in osteoblasts by wall-shear stress but not mechanical strain," J. Am. Physiol. 273, E751-E758 (1997).

20. D. Kasper et al., "Proliferation of human-derived osteoblast-like cells depends on the cycle number and frequency of uniaxial strain," J. Biomech. 35, 873-880 (2002).

21. H. Atmani et al., "Phenotypic effects of continuous or discontinuous treatment with dexamethasone and/or calcitriol on osteoblasts differentiated from rat bone marrow stromal cells," J. Cell. Biochem. 85, 640650 (2002)

22. W. Chen et al., "Multiphoton imaging and quantitative analysis of collagen production by chondrogenic human mesenchymal stem cells cultured in chitosan scaffold," Tissue Eng. 16, 913-920 (2010).

23. R. M. Delaine-Smith, S. MacNeil, and G. C. Reilly, "Matrix production and collagen structure are enhanced in two types of osteogenic progenitor cells by a simple fluid shear stress stimulus," Eur. Cells Mater. 24, 162-174 (2012).

24. F. Toki et al., "Second harmonic generation reveals collagen fibril remodeling in fibroblast-populated collagen gels," Cell Struct. Funct. 38, 229-238 (2013).

Katsuya Sato is an associate professor at Tokushima University. $\mathrm{He}$ received his $B E$ and $M E$ degrees in mechanical engineering from Kobe University in 2000 and 2002, respectively, and his $\mathrm{PhD}$ in engineering from Kobe University in 2005. His current research interests include cell biomechanics, mechanobiology, MEMS, and bone regenerative medicine. He is a member of JSME, JSMBE, and BSJ.
Oki Matsubara is a master course student at Tokushima University. $\mathrm{He}$ received his $\mathrm{BE}$ degree in mechanical engineering from Tokushima University in 2014. His current research interests include cell biomechanics, biomedical optics, and bone regenerative medicine. He is a member of JSME.

Eiji Hase received his BS, MS, and PhD degrees in engineering from Tokushima University, Japan, in 2012, 2014, and 2017, respectively. From 2014 to 2017, he was a research associate with JST, ERATO, MINOSHIMA Intelligent Optical Synthesizer Project. Since 2017, he has been a research scientist at Japan Synchrotron Radiation Research Institute, Japan, and a visiting associate professor at Tokushima University, Japan. He is a member of SPIE.

Takeo Minamikawa received his A.E. from Ibaraki National College of Technology, Japan, in 2004, received his BE, ME, and PhD from Osaka University, Japan, in 2006, 2008, and 2010, respectively. He is a JSPS Research Fellowship for Young Scientists from 2011 to 2013. $\mathrm{He}$ is an assistant professor from 2013 to 2015 , and visiting associate professor from 2015 in Kyoto Prefectural University of Medicine. He is an associate professor in Tokushima University from 2015. He is a JST-PRESTO researcher from 2017. He is a regular member of SPIE.

Takeshi Yasui is a professor at Tokushima University. He received his first $\mathrm{PhD}$ in engineering from the University of Tokushima, Tokushima, Japan, in 1997, and his second PhD in medical science from Nara Medical University, Yagi, Japan, in 2013. He is the author of more than 90 journal papers and has written three book chapters. His current research interests include $\mathrm{THz}$ instrumentation and metrology, second-harmonic-generation microscopy, and optical frequency comb. He is a member of SPIE. 